\title{
MISE EN ÉVIDENCE DE LA REPRODUCTION NATURELLE DU SAUMON ATLANTIQUE (SALMO SALAR L.) DANS UN AFFLUENT FRANÇAIS DU RHIN PAR ANALYSE GÉNÉTIQUE D'OEUFS PRÉLEVÉS DANS DES FRAYERES.
}

\author{
C.R. LARGIADÈR (1), R. GUYOMARD (1), P. ROCHE (2).
}

(1) Laboratoire de génétique des poissons, INRA, 78352 Jouy-en-Josas, France.

(2) Conseil Supérieur de la Pêche, Délégation régionale Champagne-Ardenne, AlsaceLorraine, 18 rue de Nomeny, 57158 Montigny-lès-Metz, France.

\section{RÉSUMÉ}

En utilisant la réaction de polymérisation en chaîne, nous avons pu amplifier des régions spécifiques des ADN nucléaire (région conservée des gènes du rARN 5S) et mitochondrial (région ND-5/6) extraits d'oeufs prélevés sur quatre frayères de salmonidés dans un tributaire du Rhin. Trois frayères abritaient des descendances de saumon atlantique et la quatrième une descendance de truite commune. Ces résultats prouvent que les techniques de biologie moléculaire permettent une identification des espèces de salmonidés à des stades très précoces de développement, et que les programmes de réintroduction de saumon atlantique dans le bassin du Rhin se sont traduits par une reprise de la reproduction naturelle du saumon atlantique dans la partie française de ce bassin.

Mots-clés : saumon atlantique, Rhin, migration, reproduction, repeuplement, génétique.

\section{EVIDENCE FOR NATURAL REPRODUCTION OF ATLANTIC SALMON (SALMO SALAR L.) IN A FRENCH TRIBUTARY OF THE RHINE BASED ON GENETIC ANALYSIS OF EGGS COLLECTED FROM REDDS.}

\begin{abstract}
By means of the polymerase chain reaction, we were able to amplify specific regions of the nuclear (conserved region of the 5S rRNA genes) and mitochondrial DNA (ND-5/6 segment) extracted from eggs that had been collected from four salmonid redds in a tributary of the Rhine. Three redds were identified as Atlantic salmon redds and one as brown trout redd. These results show that molecular methods allow an identification of salmonid species at very early stages of development, and that the re-introduction programmes of Atlantic salmon in the Rhine basin resulted in a re-establishment of natural reproduction of Atlantic salmon in the French part of this basin.
\end{abstract}

Key-words : Atlantic salmon, Rhine, migration, reproduction, stocking, genetics. 


\section{INTRODUCTION}

Le saumon atlantique (Salmo salar L.), très abondant dans le Rhin au siècle dernier et disparu dans les années 1950 à la suite de la construction de barrages et de l'accroissement de la pollution (de GROOT, 1989 ; ROCHE, 1990), fait l'objet d'un programme de réintroduction coordonné par la Commission Internationale pour la Protection du Rhin. Les premières captures de saumons adultes ont eu lieu aux Pays-Bas et en Allemagne à partir de 1990, principalement dans un affluent du Rhin moyen : la Sieg, où des frayères de saumons ont par la suite été découvertes et où la reproduction naturelle a été prouvée par analyse génétique d'alevins vésiculés prélevés in situ (LEHMANN et al., 1995).

Dans la partie française du bassin et dans le Rhin franco-allemand, les introductions d'alevins de saumon, principalement originaires de cours d'eau français, ont débuté en 1991 mais n'ont porté sur des nombres significatifs qu'à partir de 1992. Des pêches à l'électricité à l'aval immédiat du barrage d'Iffezheim, situé à $670 \mathrm{~km}$ de la mer et premier obstacle dans le cours principal du Rhin depuis l'estuaire, ont permis de capturer les premiers saumons adultes en 1995 (ROCHE et al., 1996). Un saumon a été capturé en juillet, et huit autres en octobre et novembre. Leur âge, déterminé à partir de la lecture d'écailles, était de un à deux ans d'eau douce et un an de mer. Six saumons ont été conservés en pisciculture et trois autres ont été relâchés dans la Bruche, affluent de la rivière ill, elle-même principal affluent du Rhin en Alsace. Ce cours d'eau possède un nombre important de sites potentiels de reproduction et son tronçon aval est relativement accessible depuis le barrage d'Iffezheim (ROCHE, 1994). Dans cet article, nous présentons une méthode moléculaire permettant de déterminer l'espèce de salmonidés à des stades très précoces de développement. Nous appliquons cette méthode à des oeufs provenant de frayères localisées sur la Bruche et susceptibles de résulter de reproduction naturelle de saumon atlantique.

\section{MATÉRIEL ET MÉTHODES}

Une recherche de frayères dans la partie aval de la Bruche a été faite sur un tronçon de $30 \mathrm{~km}$ situé entre la confluence avec l'ill et le barrage infranchissable d'Avolsheim. Sept frayères de salmonidés ont été localisées. Leurs dimensions étaient comprises entre 3 et $5 \mathrm{~m}$ de longueur, et 1 à $2 \mathrm{~m}$ de largeur. Toutes les frayères étaient situées dans une zone d'accélération du courant, à l'aval d'une fosse et à l'amont immédiat d'un radier, à une profondeur de $0,2 \mathrm{~m}$ à $0,5 \mathrm{~m}$ en étiage.

Des oeufs ont été prélevés dans quatre frayères (figure 1): Eckbolsheim E1 (un oeuf), Eckbolsheim E2 (cinq oeufs), Eckbolsheim E4 (cinq oeufs) et Holtzheim H1 (neuf oeufs), pour déterminer par analyse génétique s'ils provenaient de reproduction de truite commune (Salmo trutta L.) ou de saumon atlantique. Les oeufs prélevés étaient à un stade correspondant à 120 degrés-jours environ.

L'ADN total a été extrait de chaque oeuf selon la seconde méthode décrite dans ESTOUP et al. (1996). Deux techniques, basées sur l'amplification en chaîne par polymérisation (PCR) de fragments spécifiques d'ADN, ont été utilisées pour déterminer à quelle espèce appartenaient les différents échantillons d'oeufs. La première technique, développée par PENDAS et al. (1995), a consisté à amplifier un fragment spécifique du gène codant pour la sous-unité $5 S$ de l'ARN ribosomique. Chez les eucaryotes supérieurs, ce gène est présent en un grand nombre de copies s'enchaînant les unes à la suite des autres (répétitions en tandem). L'amplification produit un fragment d'une taille de 260 paires de bases $(\mathrm{pb})$ chez le saumon atlantique et de $280 \mathrm{pb}$ chez la truite commune. La différence de taille est caractéristique de chaque espèce, puisque PENDAS et al. (1995) l'ont observée de façon systématique en comparant plusieurs dizaines de truites et de saumons, et est facilement mise en évidence par électrophorèse en 


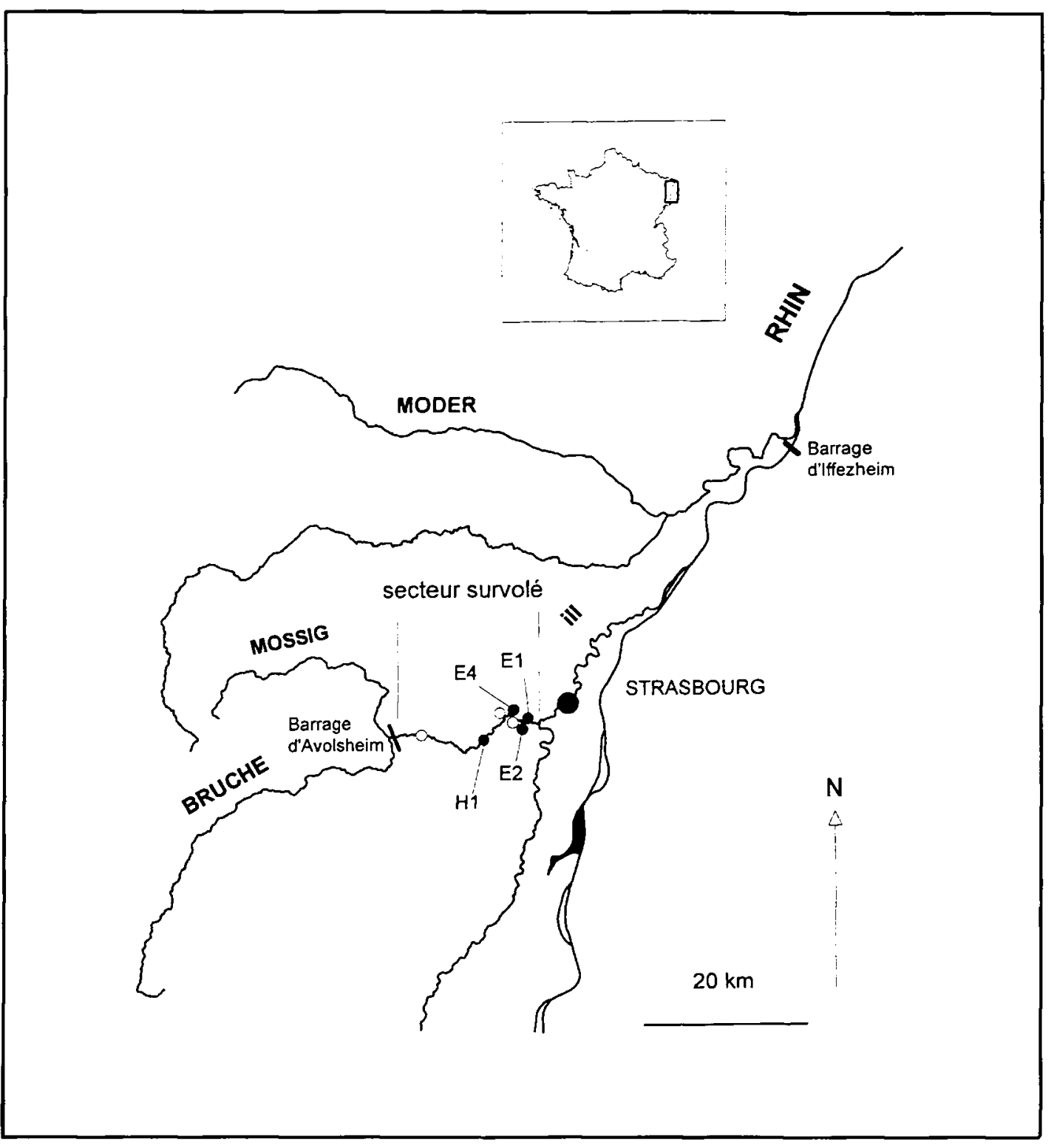

Figure 1 : Sites géographiques des frayères de saimonidés détectées (cercles). E1, E2, E4 et H1 : frayères analysées génétiquement.

Figure 1 : Geographic locations of detected salmonid redds (circles). E1, E2, E4 and H1 : redds analysed genetically.

minigel d'agarose. Cette méthode présente, en outre, l'avantage d'amplifier un fragment spécifique, non pas d'un seul locus, mais de plusieurs dizaines de copies du gène répété, ce qui accroît la puissance d'analyse. L'amplification du fragment de gène $5 \mathrm{~S}$ rDNA a été obtenue avec les amorces de PENDAS et al. (1995), à l'aide d'un thermocycleur PTC100 (MJ Research, USA). Chaque réaction de PCR a été réalisée dans $12,5 \mu$ l contenant $2 \mu \mathrm{l}$ d'ADN, du MgCl2 à la concentration de $1.2 \mathrm{mM}$, chaque dNTP à $60 \mu \mathrm{M}$, les deux amorces à $400 \mathrm{nM}, 20 \mu \mathrm{g} / \mathrm{ml}$ d'albumine sérique bovine (BSA), $1.25 \mu \mathrm{l}$ de tampon de réaction Promega concentré 10 fois et 
0,25 unité de Taq polymérase (Promega). Le profil thermique d'amplification comportait une dénaturation à $95^{\circ} \mathrm{C}$ pendant $6 \mathrm{mn}$, suivie de cinq cycles d'une mn à $95^{\circ} \mathrm{C}, 30 \mathrm{~s}$ à $63^{\circ} \mathrm{C}$ et $2 \mathrm{mn}$ à $72{ }^{\circ} \mathrm{C}$, puis 27 cycles de $30 \mathrm{~s}$ à $94^{\circ} \mathrm{C}, 30 \mathrm{~s}$ à $63^{\circ} \mathrm{C}$ et $2 \mathrm{mn}$ à $72^{\circ} \mathrm{C}$, enfin une étape d'élongation de $5 \mathrm{mn}$ à $72^{\circ} \mathrm{C}$. La seconde méthode était basée sur l'analyse du polymorphisme de longueur de fragment de restriction (PLFR) observé après digestion de la région ND-5/6 de I'ADN mitochondrial par Hinf I. L'amplification de la région ND-5/6, d'une longueur d'environ 2.5 $\mathrm{kb}$, a été obtenue avec les amorces décrites par CRONIN et al. (1993). La réaction de PCR a été réalisée dans un volume total de $50 \mu \mathrm{l}$ contenant $3 \mu \mathrm{l}$ d'ADN, du $\mathrm{MgCl} 2$ à $1.5 \mathrm{mM}$, chaque dNTP à $240 \mu \mathrm{M}$, chaque amorce à $200 \mathrm{nM}, 10 \mu \mathrm{g} / \mathrm{ml}$ de BSA, $5 \mu$ de tampon de réaction Promega concentré 10 fois et 2 unités de Taq polymérase (Promega). Les conditions de PCR étaient les suivantes : $3 \mathrm{mn}$ à $95^{\circ} \mathrm{C}$, cinq cycles d'une $\mathrm{mn}$ à $94^{\circ} \mathrm{C}, 30$ s à $52^{\circ} \mathrm{C}$ et $3 \mathrm{mn}$ à $72^{\circ} \mathrm{C}$, puis 27 cycles d'une $\mathrm{mn}$ à $94^{\circ} \mathrm{C}, 30 \mathrm{~s}$ à $50^{\circ} \mathrm{C}$ et $3 \mathrm{mn}$ à $72^{\circ} \mathrm{C}$, enfin une étape d'élongation de $10 \mathrm{mn}$ à $72{ }^{\circ} \mathrm{C}$. La digestion de 3 à $8 \mu \mathrm{l}$ de produits de PCR par Hinf I a été réalisée selon les recommandations du fournisseur (Amersham). Les électrophorèses ont été effectuées sur gels d'agarose et les fragments d'ADN révélés en éthidium bromide.

\section{RÉSULTATS ET DISCUSSION}

Les régions rARN-5S et ND-5/6 ont pu être amplifiées spécifiquement pour tous les oeufs échantillonnés. Des ADN de référence de trois espèces de salmonidés (truite arc-en-ciel, truite commune et saumon atlantique d'Europe et du Canada) ont également été amplifiés. La taille des fragments rARN-5S obtenus chez chacun des témoins est conforme aux résultats de PENDAS et al. (1995). Par comparaison de la taille des produits de PCR des ADN de référence et des oeufs (figure 2A), il apparait clairement que trois des frayères (Eckbolsheim E1, E2 et E4) correspondent à une reproduction de saumon atlantique et Holtzheim à une frayère de truite commune. Les profils de restriction de la région ND-5/6 confirment cette conclusion. Les oeufs de frayères Eckbolsheim E1, E2 et E4 donnent des haplotypes mitochondriaux identiques à ceux trouvés chez le saumon atlantique (figure 2B) et totalement différents de ceux observés jusqu'à présent chez un grand nombre d'échantillons de truites communes provenant d'une vaste zone géographique (HANSEN et LOESCHKE, 1996 ; HANSEN et al., sous presse ; GUYOMARD et LARGIADĖR, données non publiées). La figure 2B montre aussi que l'haplotype observé pour ces frayères se distingue très nettement de celui de la truite arc-en-ciel. En revanche, les oeufs de la frayère d'Holtzheim présentent un haplotype fréquemment révélé dans les populations atlantiques domestiques et naturelles de truite commune.

Ces résultats confirment qu'il est possible de disposer de méthodes moléculaires relativement simples pour déterminer rapidement l'espèce d'origine d'oeufs de saumon ou de truite commune, à des stades précoces de développement. Appliquées à l'analyse d'oeufs provenant de frayères de la Bruche, elles nous ont permis d'identifier sans ambiguité l'existence de sites de reproduction naturelle de saumons atlantiques dans ce cours d'eau, fait sans précédent depuis plusieurs décennies. Cette identification est indispensable pour conduire des opérations appropriées de soutien et de protection des populations fondatrices vulnérables, et assurer le succès des programmes de réintroduction.

L'ADN mitochondrial ne constitue pas uniquement un marqueur supplémentaire pour l'identification de l'espèce, mais peut aussi apporter une information importante en cas d'hybridation entre la truite commune et le saumon atlantique. En effet, compte tenu du mode d'hérédité strictement maternel de I'ADN mitochondrial, l'analyse conjointe de marqueurs mitochondriaux et nucléaires permet de déterminer le sens de l'hybridation. L'existence d'hybridation entre les deux espèces est régulièrement mise en évidence sur l'ensemble de leur zone de sympatrie (VERSPOOR et HAMMAR, 1991). Les causes et conséquences de ce phénomène sont inconnues. II ne peut être exclu que de faibles densités de population (comme cela peut être le cas dans les programmes de restauration d'une espèce) ou l'utilisation de souches inadaptées soient des facteurs-clés favorisant cette hybridation. 

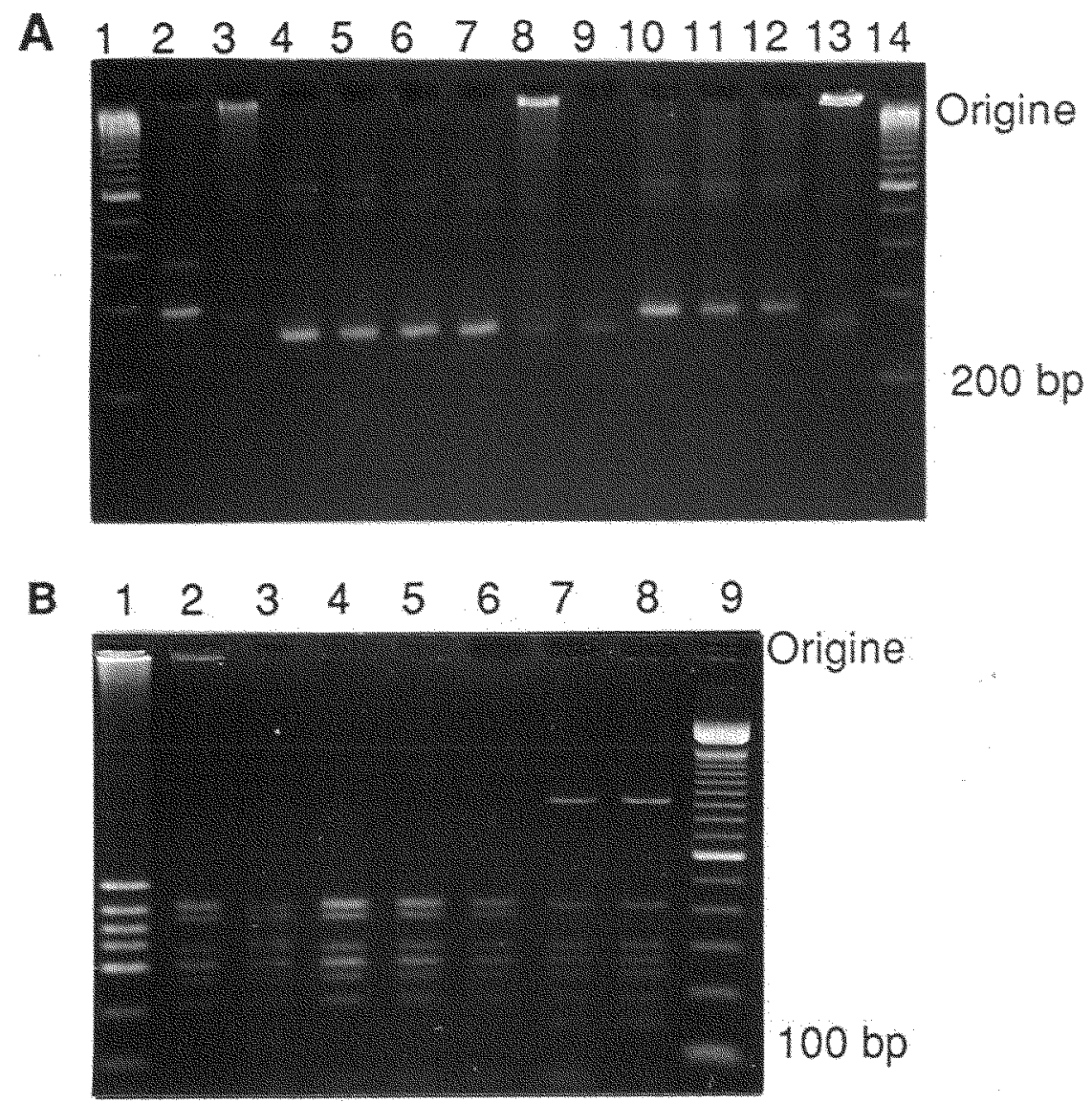

Figure 2 : Électrophorèse, en gel d'agarose, de produits d'amplification par PCR à partir d'ADN extraits d'oeufs de salmonidés.

(A) : région rARN-5S; 1 et 14 : marqueur de taille (échelle de $100 \mathrm{pb}$; la bande correspondant à 200 pb est indiquée) ; 2 : échantillon de référence de truite arc-en-ciel ; 3 et 12 : échantillons de référence de truite commune; 8 et 13 : échantillons de référence de saumon atlantique ; 4 et 5 : oeufs de la frayère E4; 6 et 7 : oeufs de la frayère $E 2$; 9 : oeuf de la frayère $E 1 ; 10$ et 11 : oeufs de la frayère $H 1$.

(B) : région ND-5/6 digérée avec Hinf I ; 1 : échantillon de référence de truite arc-en-ciel ; 2 et 3 : échantillons de référence de saumon atlantique, respectivement canadien et européen ; 4 : oeufs de la frayère $E 4 ; 5$ : oeuf de la frayère E2 ; 6 : oeuf de la frayère E1; 7 : oeuf de la frayère $\mathrm{H} 1 ; 8$ : échantillon de référence de truite commune ; 9 : marqueur de taille (échelle de $100 \mathrm{bp}$; la bande correspondant à $100 \mathrm{pb}$ est indiquée).

Figure 2 : Agarose gel electrophoresis of amplification products from DNA extracted from salmonid eggs.

(A) : 5S-rRNA region ; 1 and 14 : size marker (100 bp ladder ; the 200 bp band is indicated) ; 2 : reference sample of rainbow trout ; 3 and 12 : reference samples of brown trout ; 8 and 13 : reference samples of Atlantic salmon ; 4 and 5 : eggs from redd E4; 6 and 7 : eggs from redd $E 2 ; 9$ : egg from redd $E 1 ; 10$ and 11 : eggs from redd $H 1$.

(B) : ND-5/6 region digested with Hinf I ; 1 : reference sample of rainbow trout ; 2 and 3 : reference samples of European and Canadian Atlantic salmon, respectively ; 4 : eggs from redd $E 4 ; 5$ : egg from redd E2 ; 6 : egg from redd $E 1 ; 7$ : egg from redd $H 1 ; 8$ : reference sample of brown trout ; 9 : size marker (100 bp ladder; the 100 bp band is indicated). 


\section{REMERCIEMENTS}

C.R. LARGIADĖR est boursier du fonds national suisse de la recherche scientifique. Les travaux de laboratoire ont été partiellement financés par l'Action Incitative Programmée INRABureau des Ressources Génétiques $n^{\circ}$ P00060 "Structuration génétique des populations naturelles". Les auteurs remercient A. ESTOUP et F. KRIEG (INRA, Laboratoire de génétique des poissons) pour leurs conseils techniques.

\section{BIBLIOGRAPHIE}

CRONIN M.A., SPEARMAN W.J., WILMOT R.L., PATTON J.C., BICKHAM J.W., 1993. Mitochondrial DNA variation in Chinook (Oncorhynchus tshawytscha) and Chum salmon $(O$. keta) detected by restriction enzyme analysis of polymerase chain reaction (PCR) products. Canadian Journal of Fisheries and Aquatic Sciences, 50, 708-715.

ESTOUP A., LARGIADĖR C.R., PERROT E., CHOURROUT D., 1996. Rapid one-tube DNA extraction for reliable PCR detection of fish polymorphic markers and transgenes. Molecular Marine Biology and Biotechnology, 5 (3), 295-298.

GROOT (de) S.J., 1989. Literature survey into the possibility of restocking the River Rhine and its tributaries with Atlantic salmon (Salmo salar). Publication and reports Vol. 1989-11 of the project "Ecological Rehabilitation of the River Rhine", RIVO, ljmuiden, the Netherlands, 56 p. + annexes.

HANSEN M.M., LOESCHKE V., 1996. Genetic differentiation among Danish brown trout populations, as detected by RFLP analysis of PCR amplified mitochondrial DNA segments. Journal of Fish Biology, 48, 422-436.

HANSEN M.M., MENSBERG K.L.D., RASMUSSEN G., SIMONSEN V., sous presse. Genetic variation within and among Danish brown trout (Salmo trutta) hatchery strains, assessed by PCR-RFLP analysis of mitochondrial DNA segments. Aquaculture.

LEHMANN J., SCHENK M., STÜRENBERG F., SCHREIBER A., 1995. Natural reproduction or recolonizing Atlantic salmon, Salmo salar, in the Rhenanian drainage system (NordrheinWestfalen, Germany). Naturwissenschaften, 82, 92-93.

PENDAS A.M., MORAN P., MARTINEZ J.L., GARCIA-VAZQUEZ E., 1995. Applications of $5 S$ rDNA in Atlantic salmon, brown trout, and in Atlantic salmon $x$ brown trout hybrid identification. Molecular Ecology, 4, 275-276.

ROCHE P., 1990. Le saumon du Rhin : données historiques. Rapport CSP Metz-Ministère de l'environnement-DRAE Alsace, $65 \mathrm{p}$.

ROCHE P., 1994. Habitat availability and carrying capacity in the French part of the Rhine for Atlantic salmon (Salmo salar L.). Wat. Sci. Tech., 29 (3), 257-265.

ROCHE P., EDEL G., GERLIER M., 1996. Premières captures de saumons (Salmo salar L.) dans le Rhin franco-allemand et mise en évidence de frayères dans la Bruche. Rapport CSP Metz, $7 \mathrm{p}$.

VERSPOOR E., HAMMAR J., 1991. Introgressive hybridization in fishes : the biochemical evidence. Journal of Fish Biology, 39 (Supplement A), 309-334. 